\title{
Structure of New Carotenoids with a 3,4-Dihydroxy- $\beta$-End Group from the Oyster Crassostrea gigas
}

\author{
Takashi MaокA, ${ }^{* a}$ Yasuhiro Fujiwara, ${ }^{b}$ Keiji Hashimoto, ${ }^{c}$ and Naoshige AкIмото ${ }^{d}$ \\ ${ }^{a}$ Research Institute for Production Development; 15 Shimogamo-morimoto-cho, Sakyo-ku, Kyoto 606-0805, Japan: ${ }^{b}$ Kyoto \\ Pharmaceutical University; 5 Nakauchi-cho, Misasagi, Yamashina-ku, Kyoto 607-8414, Japan: ${ }^{c}$ Nagahama Institute of \\ Bio-sciences and Technology; Tamura-cho, Nagahama, Shiga 526-0829, Japan: and ${ }^{d}$ Graduate School of Pharmaceutical \\ Sciences; Kyoto University; Yoshida-shimoadachi-cho, Sakyo-ku, Kyoto 606-8501, Japan. \\ Received May 23, 2005; accepted June 7, 2005; published online June 13, 2005
}

\begin{abstract}
Three new carotenoids with a 3,4-dihydroxy- $\beta$-end group were isolated from the oyster Crassostrea gigas. These structures were determined to be $3,4,3^{\prime}, 8^{\prime}$-tetrahydroxy- $\beta$, $\kappa$-caroten- $6^{\prime}$-one (1), 3,4-dihydroxy-3', $6^{\prime}$-epoxy$1^{\prime}, 2^{\prime}, 5^{\prime}, 6^{\prime} 7^{\prime}, 8^{\prime}$-hexahydro-6'-methyl-16'-nor- $\beta, \varphi$-carotene-1', $\mathbf{8}^{\prime}$-dione (2), and 3,6 -epoxy-5,3', $4^{\prime}$-trihydroxy$12^{\prime}, 13^{\prime}, 20^{\prime}$-trinor- $\beta, \beta$-caroten-19,11-olide (3) based on chemical and spectral data.
\end{abstract}

Key words $\quad$ oyster; Crassostrea gigas; carotenoid; ${ }^{1} \mathrm{H}-\mathrm{NMR}$; FAB-MS

Marine animals contain various carotenoids which have structural variety. ${ }^{1)}$ Some of them exhibit antioxidative, ${ }^{2)}$ anti-tumore, and anti-carcinogenic activites. ${ }^{3-5)}$ Shellfish, which are important seafood in Japan, contain several structurally unique carotenoids. ${ }^{1)}$ This prompted us to search for new carotenoids from shellfish.

Mytiloxanthin, isomytiloxanthin from Mytilus edulis, ${ }^{6,7)}$ diatoxanthin, alloxanthin, and pectinolone from Pectene maximus $^{8)}$ and Patinopectene yessoensis, ${ }^{9)}$ pectenol $\mathrm{A}$ and $\mathrm{B}^{10)}$ and $8^{\prime}$-apoalloxanthinal ${ }^{11)}$ from Mytilus coruscus, mactraxanthin from Mactra chinensis, ${ }^{12)}$ crassostreaxanthin A and B from Crassostrea gigas,${ }^{13)}$ and a series of carotenoids with a 5,6-dihydro- $\beta$-end group from Fushinus perplexus, ${ }^{14)}$ have been reported as principal carotenoids in marine shellfish.

In the course of studies of the carotenoids in shellfish, previously we reported the isolation and structural elucidation of five new carotenoids having a unique structure from the oyster Crassostrea gigas. ${ }^{15)}$ Recently, we isolated a series of new carotenoids with a 3,4-dihydroxy- $\beta$-end group as minor components from the same species. This paper reports the isolation and structural elucidation of these new carotenoids based on chemical and spectral data.

The $\mathrm{Me}_{2} \mathrm{CO}$ extract of the edible part of $C$. gigas, was chromatographed on silica gel using an increasing percentage of $\mathrm{Me}_{2} \mathrm{CO}$ in hexane. The fraction eluted with $\mathrm{Me}_{2} \mathrm{CO}$-hexane $(6: 4)$ was subjected to HPLC on silica gel with $\mathrm{Me}_{2} \mathrm{CO}$-hexane $(6: 4)$ and then on ODS with $\mathrm{CHCl}_{3}-\mathrm{MeCN}(1: 9)$ to yield $\mathbf{1}(0.3 \mathrm{mg}), 2(0.3 \mathrm{mg})$, and $\mathbf{3}$ (0.2 mg).

Compound 1 showed absorption maximum at $470 \mathrm{~nm}$. The molecular formula of 1 was determined to be $\mathrm{C}_{40} \mathrm{H}_{56} \mathrm{O}_{5}$ by HR-FAB-MS. The presence of three secondary hydroxy groups in $\mathbf{1}$ was consistent with the formation of a triacetate. Treatment of 1 with $5 \% \mathrm{KOH} / \mathrm{C}_{2} \mathrm{H}_{5} \mathrm{OH}$ showed reversible hypsochromic shift $15 \mathrm{~nm}$ and reduction of 1 with $\mathrm{NaBH}_{4}$ gave a pentaol, having absorption maxima at 405,429 , and $465 \mathrm{~nm}$. These visible spectral properties were in agreement with the presence of a mytiloxanthin type chromophore ${ }^{6,16)}$ in 1. The ${ }^{1} \mathrm{H}-\mathrm{NMR}$ data for 1 indicated the presence of a 3,4(cis)-dihydroxy- $\beta$-end group (C-1 to $\mathrm{C}-6$ including $\mathrm{C}-16,17$, and 18$),{ }^{10,17)}$ an 6-oxo-3-hydroxy- $\kappa$-end group $\left(\mathrm{C}-1^{\prime}\right.$ to $\mathrm{C}-6^{\prime}$ including $\mathrm{C}-16^{\prime}, 17^{\prime}$, and $18^{\prime}$ ), and a polyene chain contain- ing an enolic group $\left(\delta 16.3, \mathrm{~s}, \mathrm{H}-8^{\prime}\right) .{ }^{6,7,16)}$ The ${ }^{1} \mathrm{H}-{ }^{1} \mathrm{H}$ connectivities of $\mathrm{H}-2$ to $\mathrm{H} 4, \mathrm{H} 2^{\prime}$ to $\mathrm{H}-4^{\prime}$, and olefinic protons were confirmed by decoupling experiments. The cis configuration of the 3,4-glycol group was revealed by a coupling constant between $\mathrm{H}-3$ and $\mathrm{H}-4$ of $3.5 \mathrm{~Hz} \cdot{ }^{10,17)}$ Therefore, the structure of 1 was determined to be 3,4,3', $8^{\prime}$-tetrahydroxy$\beta, \kappa$-caroten- $6^{\prime}$-one. The shape and magnitude of CD spectrum of 1 was similar to that of capsanthin, $\left(3 R, 3^{\prime} S, 5^{\prime} R\right)$ $3,3^{\prime}$-dihydroxy- $\beta$, $\kappa$-caroten- $6^{\prime}$-one. ${ }^{18)}$ These results suggested that $\mathbf{1}$ had the same chiralitis at $\mathrm{C}-3$ and $\mathrm{C}-5^{\prime}$ as those of capsanthin. The $3^{\prime} S$ chiraly was proposed from biosynthetic consideration. Therefore, on the basis of CD data, relative stereochemistry of 3,4-dihydroxy group, and biosynthetic considerations, a $\left(3 S, 4 R, 3^{\prime} S, 5^{\prime} R\right)$ configuration was postulated for $\mathbf{1}$.

Compound 2 showed absorption maxima at 450 and $470 \mathrm{~nm}$. The molecular formula of 2 was determined to be $\mathrm{C}_{40} \mathrm{H}_{56} \mathrm{O}_{5}$ by HR-FAB-MS. The presence of two secondary hydroxy groups in $\mathbf{2}$ was consistent with the formation of a diacetate. Reduction of $\mathbf{2}$ with $\mathrm{NaBH}_{4}$ gave a tetrol, having absorption maxima at 405,429 , and $465 \mathrm{~nm}$, suggesting the presence of a conjugated carbonyl group in a polyene chian. ${ }^{19)}$ The ${ }^{1} \mathrm{H}$ chemical shifts and spin-couplings of $\mathrm{H} 2$ to $\mathrm{H} 20$ and $\mathrm{H}^{\prime}{ }^{\prime}$ to $\mathrm{H}_{2} \mathrm{O}^{\prime}$ in $\mathbf{2}$ were almost identical with those of $\left(3 S, 4 R, 3^{\prime} S, 4^{\prime} R\right)$-curastaxanthin ${ }^{20)}$ and crassestreaxanthin $\mathrm{A},{ }^{13)}$ respectively. The cis configuration of 3,4-glycol was also confirmed by coupling constant of $3.5 \mathrm{~Hz} .^{10,17)}$ The ${ }^{1} \mathrm{H}-{ }^{1} \mathrm{H}$ connectivities of $\mathrm{H}-2$ to $\mathrm{H} 4, \mathrm{H}^{\prime}$ to $\mathrm{H}-18^{\prime}$, and olefinic protons were also confirmed by decoupling experiments. Therefore, the structure of $\mathbf{2}$ was determined to be 3,4-dihydroxy-3', $6^{\prime}$-epoxy-1' $2^{\prime}, 5^{\prime}, 6^{\prime} 7^{\prime}, 8^{\prime}$-hexahydro-6' methyl-16' $6^{\prime}$-nor- $\beta, \varphi$-carotene-1', $8^{\prime}$-dione. The $(3 S, 4 R)$ configuration was postulated for $\mathbf{2}$ by $\mathrm{CD}$ spectrum comparison with $\left(3 S, 4 R, 3^{\prime} S, 4^{\prime} R\right)$-curastaxanthin ${ }^{20)}$ and biosynthetic consideration.

Compound 3 showed absorption maxima at 455 and $475 \mathrm{~nm}$. The molecular formula of $\mathbf{3}$ was determined to be $\mathrm{C}_{37} \mathrm{H}_{48} \mathrm{O}_{6}$ by HR-FAB-MS. The characteristic ${ }^{1} \mathrm{H}-\mathrm{NMR}$ signals of olefinic protons suggested the presence of a butenolide group in the polyene chain. ${ }^{17)}$ The presence of two secondary hydroxy groups in $\mathbf{3}$ was confirmed by acetylation. As well as compounds 1 and $\mathbf{2}$, the presence of a 3,4-(cis)-di- 

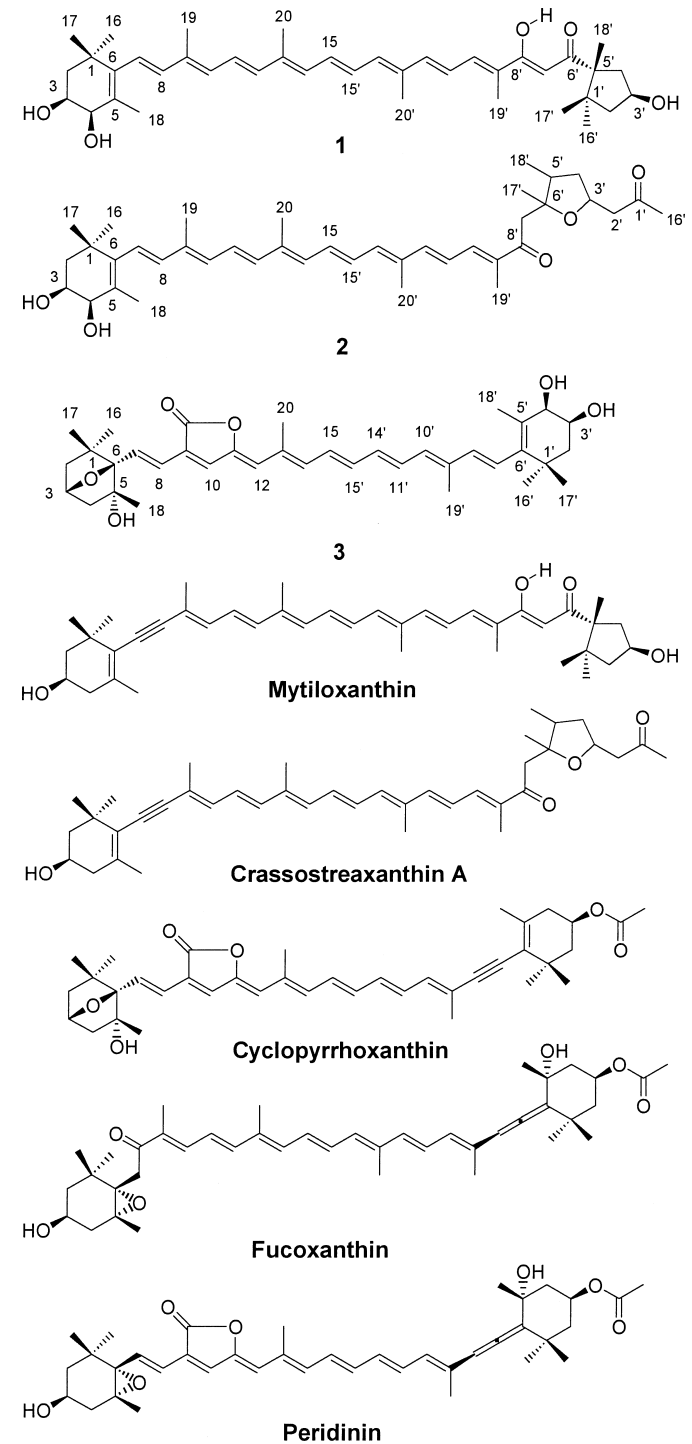

hydroxy- $\beta$-end group in $\mathbf{3}$ was revealed by ${ }^{1} \mathrm{H}$-NMR data. The ${ }^{1} \mathrm{H}-\mathrm{NMR}$ signals of the remaining end group ( $\mathrm{H}-2$ to $\mathrm{H}-$ 4, H-16, 17, and H-18) were similar to those of cycloviolaxanthin ${ }^{21)}$ suggesting presence of the $(3 S, 5 R, 6 R)-3,6$-epoxy5,6 -dihydro-5-hydroxy- $\beta$-end group. Furthermore, the ${ }^{1} \mathrm{H}$ chemical shifts and the spin-couplings of $\mathrm{H} 2$ to $\mathrm{H} 8$ including methyl groups H16, 17, and 18 were identical with those of cyclopyrrhoxanhin, a corresponding 3,6-epoxide of pyrrhoxanthin isolated from Corbicula japonica. ${ }^{22)}$ Thus the structure 3,6-epoxy-5,3', $4^{\prime}$-trihydroxy-12',13',20'-trinor- $\beta, \beta$ caroten-19,11-olide was postulated for 3 . The ${ }^{1} \mathrm{H}-{ }^{1} \mathrm{H}$ connectivities of $\mathrm{H}-2$ to $\mathrm{H} 4, \mathrm{H} 2^{\prime}$ to $\mathrm{H}-4^{\prime}$, and olefinic protons were also confirmed by decoupling experiments. The $\left(3 S, 5 R, 6 R, 3^{\prime} S, 4^{\prime} R\right)$ chirality was tentatively proposed for $\mathbf{3}$ by biosynthetic consideration.

In the present study, three new carotenoids, 1, 2, and $\mathbf{3}$, having a 3,4-dihydroxy- $\beta$-end group were isolated from the oyster $C$. gigas in addition to 22 carotenoids that had been isolated previously. ${ }^{13,15)}$ In general, animals do not synthesize carotenoids de novo and those found in animals are either directly accumulated from food or partly modified through metabolic reactions. ${ }^{23)}$ The food sources of oyster are phytoplankton such as diatom and dinoflagellate. The major carotenoids in diatom and dinoflagellate are fucoxanthin and peridinin, respectively. ${ }^{23)}$ It was reported that mitiloxanthin and crassestreaxanthin A were metabolites of fucoxanthin, which was accumulated from dietal diatom, in shellfish. ${ }^{13,15,16,23)}$ Compounds $\mathbf{1}$ and $\mathbf{2}$ might be derived from mytiloxanthin and crassestreaxanthin A, respectively. Compound 3 is a trinorcarotenoid having $\mathrm{C}_{37}$ skeletne and was a likely metabolite of peridinin, which was accumulated from dietal dinoflagellate.

In the present paper, the IUPAC carotenoid nomenclature was based on the new rules described in the "Carotenoids Handbook.",24)

\section{Experimental}

Apparatus The UV-visible (vis) spectra were recorded with a Shimadzu UV-240 spectrophotometer in diethyl ether $\left(\mathrm{Et}_{2} \mathrm{O}\right)$. The positive ion FAB-MS were recorded using a JEOL JMS-SX 102 or JMX-HX 110A mass spectrometer with $m$-nirobenzyl alcohol ( $m$-NBA) as a matrix. The ${ }^{1} \mathrm{H}$-NMR $(300 \mathrm{MHz})$ spectra were measured with a Varian XL-300 spectrometer in $\mathrm{CDCl}_{3}$ with tetramethylsilane (TMS) as an internal standard. The CD spectra were recorded in $\mathrm{Et}_{2} \mathrm{O}$ at room temperature with a JASCO J-500 spectropolarimeter. HPLC was performed on a Shimadzu LC-6AD with a Shimadzu SPD-6AV spectrophotometer set at $450 \mathrm{~nm}$. Acetylation, trimethyl silylation, and reduction of $\mathrm{NaBH}_{4}$ were carried out using standard procedures. $^{16,25)}$

Animal Material The Oyster, C. gigas was purchased at a local fish market in January and February.

Extraction and Isolation of Carotenoids The $\mathrm{Me}_{2} \mathrm{CO}$ extract of the edible parts of C. gigas $\left(10 \mathrm{~kg}\right.$ ) was partitioned between $\mathrm{Et}_{2} \mathrm{O}$ and aqueous $\mathrm{NaCl}$. The organic layer was dried over $\mathrm{Na}_{2} \mathrm{SO}_{4}$ and then concentrated to dryness. The residue was subjected to silica gel column chromatography using an increasing percentage of $\mathrm{Me}_{2} \mathrm{CO}$ in $n$-hexane. The fraction eluted with $\mathrm{Me}_{2} \mathrm{CO}$-hexane $(6: 4)$ was subjected to a series of HPLCs on silica gel with $\mathrm{Me}_{2} \mathrm{CO}$-hexane $(6: 4)$ and then on ODS with $\mathrm{CHCl}_{3}-\mathrm{CH}_{3} \mathrm{CN}(1: 9)$ to to yield $\mathbf{1}(0.3 \mathrm{mg}), \mathbf{2}(0.3 \mathrm{mg})$, and $\mathbf{3}(0.2 \mathrm{mg})$.

Detailes of the carotenoids composition in $C$. gigas were described elsewhere. ${ }^{15)}$

Compound (1): Reddish solid. UV-vis: $\lambda_{\max }\left(\mathrm{Et}_{2} \mathrm{O}\right) 470 \mathrm{~nm},(5 \% \mathrm{KOH} /$ $\left.\mathrm{C}_{2} \mathrm{H}_{5} \mathrm{OH}\right) 455 \mathrm{~nm} .{ }^{1} \mathrm{H}-\mathrm{NMR}\left(\mathrm{CDCl}_{3}, 300 \mathrm{MHz}\right) \delta: 0.85\left(3 \mathrm{H}, \mathrm{s}, \mathrm{H}-16^{\prime}\right), 1.07$ $(3 \mathrm{H}, \mathrm{s}, \mathrm{H}-17), 1.09$ (3H, s, H-16), $1.19\left(3 \mathrm{H}, \mathrm{s}, \mathrm{H}-17^{\prime}\right), 1.35\left(3 \mathrm{H}, \mathrm{s}, \mathrm{H}-18^{\prime}\right)$, ca. $1.56(2 \mathrm{H}$, overlapped, H-2eq, H-4' $\beta), 1.68(1 \mathrm{H}, \mathrm{dd}, J=12.5,12.5 \mathrm{~Hz}, \mathrm{H}-$ $2 \mathrm{ax}), 1.72\left(1 \mathrm{H}, \mathrm{dd}, J=13.5,4.5 \mathrm{~Hz}, \mathrm{H}-2^{\prime} \beta\right), 1.90(3 \mathrm{H}, \mathrm{s}, \mathrm{H}-18), 1.98(12 \mathrm{H}, \mathrm{s}$, $\left.\mathrm{H}-19,20,19^{\prime}, 20^{\prime}\right), 2.19\left(1 \mathrm{H}, \mathrm{dd}, J=13.5,8 \mathrm{~Hz}, \mathrm{H}-2^{\prime} \alpha\right), 2.88(1 \mathrm{H}, \mathrm{dd}$, $\left.J=14.5,8.5 \mathrm{~Hz}, \mathrm{H}^{\prime} 4^{\prime} \alpha\right), 3.88(1 \mathrm{H}, \mathrm{m}, \mathrm{H}-3), 3.97(1 \mathrm{H}, \mathrm{d}, J=3.5 \mathrm{~Hz}, \mathrm{H}-4)$, $4.55\left(1 \mathrm{H}, \mathrm{m}, \mathrm{H}-3^{\prime}\right), 5.86\left(1 \mathrm{H}, \mathrm{s}, \mathrm{H}^{-} 7^{\prime}\right), 6.09(1 \mathrm{H}, \mathrm{d}, J=16 \mathrm{~Hz}, \mathrm{H}-7), 6.10$ $(1 \mathrm{H}, \mathrm{d}, J=11.5 \mathrm{~Hz}, \mathrm{H}-10), 6.17(1 \mathrm{H}, \mathrm{d}, J=16 \mathrm{~Hz}, \mathrm{H}-8), 6.28(1 \mathrm{H}, \mathrm{d}$, $J=11.5 \mathrm{~Hz}, \mathrm{H}-14), 6.36(1 \mathrm{H}, \mathrm{d}, J=15.5 \mathrm{~Hz}, \mathrm{H}-12), 6.38(1 \mathrm{H}, \mathrm{d}, J=11.5 \mathrm{~Hz}$, $\left.\mathrm{H}-14^{\prime}\right), 6.50-6.80\left(6 \mathrm{H}\right.$, overlapped, $\left.\mathrm{H}-11,15,10^{\prime}, 11^{\prime}, 12^{\prime}, 15^{\prime}\right), 16.3(1 \mathrm{H}$, s, OH-8'). HR-FAB-MS: $m / z 616.4132\left(\right.$ Calcd for $\left.\mathrm{C}_{40} \mathrm{H}_{56} \mathrm{O}_{5}, 616.4125\right)$. CD: $\left(\mathrm{Et}_{2} \mathrm{O}\right) \lambda \mathrm{nm}(\Delta \varepsilon) 235(-3.0), 239(0), 248(+2.0), 255(0), 290(-5.0), 315$ $(0), 338(+2.5), 345(0), 355(-3.0)$

Acetylation of $1(0.1 \mathrm{mg})$ with $\left(\mathrm{CH}_{3} \mathrm{CO}\right)_{2} \mathrm{O}(5 \mathrm{ml})$ in dry pyridine $(5 \mathrm{ml})$ at room temperature for $60 \mathrm{~min}$ produced a triacetate (yield 90\%) which showed molecular ion at $m / z 742$ by FAB-MS.

Reduction of $1(0.1 \mathrm{mg})$ with $\mathrm{NaBH}_{4}(5 \mathrm{mg})$ in $\mathrm{CH}_{3} \mathrm{OH}(10 \mathrm{ml})$ at room temperature for $30 \mathrm{~min}$ produced a pentaol (yield $80 \%$ ) which showed molecular ion at $m / z 618$ by FAB-MS and absorption maxima $\left(\mathrm{Et}_{2} \mathrm{O}\right)$ at 405,429 , and $465 \mathrm{~nm}$.

Compound (2): Reddish solid. UV-vis: $\lambda_{\max }\left(\mathrm{Et}_{2} \mathrm{O}\right) 450$ and $470 \mathrm{~nm} .{ }^{1} \mathrm{H}-$ NMR $\left(\mathrm{CDCl}_{3}, 300 \mathrm{MHz}\right) \delta: 0.99\left(3 \mathrm{H}, \mathrm{d}, J=7 \mathrm{~Hz}, \mathrm{H}-18^{\prime}\right), 1.07(3 \mathrm{H}, \mathrm{s}, \mathrm{H}-$ 17), 1.09 (3H, s, H-16), 1.10 (3H, s, H-17'), $1.31(1 \mathrm{H}, \mathrm{ddd}, J=12,11,10 \mathrm{~Hz}$, $\left.\mathrm{H}-4^{\prime}\right), 1.57(1 \mathrm{H}$, ddd, $J=12.5,4,1.5 \mathrm{~Hz}, \mathrm{H}-2 \mathrm{eq}), 1.68(1 \mathrm{H}, \mathrm{dd}, J=12.5$, $12.5 \mathrm{~Hz}, \mathrm{H}-2 \mathrm{ax}), 1.90$ (3H, s, H-18), 1.94 (3H, s, H-19'), 1.98 (3H, s, H-19), $1.99\left(6 \mathrm{H}, \mathrm{s}, \mathrm{H}-20,20^{\prime}\right), 2.14\left(3 \mathrm{H}, \mathrm{s}, \mathrm{H}-16^{\prime}\right), 2.17(1 \mathrm{H}, \mathrm{ddd}, J=12,7,5 \mathrm{~Hz}$, H-4' $), 2.32\left(1 \mathrm{H}\right.$, ddq, $\left.J=11,7,7 \mathrm{~Hz}, \mathrm{H}-5^{\prime}\right), 2.52\left(1 \mathrm{H}, \mathrm{dd}, J=15,5 \mathrm{~Hz}, \mathrm{H}-2^{\prime}\right)$, $2.69\left(1 \mathrm{H}, \mathrm{dd}, J=15,7 \mathrm{~Hz}, \mathrm{H}-2^{\prime}\right), 2.86\left(1 \mathrm{H}, \mathrm{d}, J=13.5 \mathrm{~Hz}, \mathrm{H}^{\prime} 7^{\prime}\right), 2.93(1 \mathrm{H}, \mathrm{d}$, $\left.J=13.5 \mathrm{~Hz}, \mathrm{H}-7^{\prime}\right), 3.88(1 \mathrm{H}, \mathrm{m}, \mathrm{H}-3), 3.97(1 \mathrm{H}, \mathrm{d}, J=3.5 \mathrm{~Hz}, \mathrm{H}-4), 4.21$ $\left(1 \mathrm{H}, \mathrm{m}, \mathrm{H}-3^{\prime}\right), 6.09(1 \mathrm{H}, \mathrm{d}, J=16 \mathrm{~Hz}, \mathrm{H}-7), 6.10(1 \mathrm{H}, \mathrm{d}, J=11.5 \mathrm{~Hz}, \mathrm{H}-10)$, $6.17(1 \mathrm{H}, \mathrm{d}, J=16 \mathrm{~Hz}, \mathrm{H}-8), 6.28(1 \mathrm{H}, \mathrm{d}, J=11.5 \mathrm{~Hz}, \mathrm{H}-14), 6.38(1 \mathrm{H}, \mathrm{d}$, $J=15.5 \mathrm{~Hz}, \mathrm{H}-12), 6.42\left(1 \mathrm{H}, \mathrm{d}, J=11.5 \mathrm{~Hz}, \mathrm{H}-14^{\prime}\right), 6.50-6.80(5 \mathrm{H}$, overlapped, H-11, 15, 11' $\left., 12^{\prime}, 15^{\prime}\right), 7.26\left(1 \mathrm{H}, \mathrm{d}, J=11 \mathrm{~Hz}, \mathrm{H}-10^{\prime}\right)$. HR-FAB- 
MS: $m / z 616.4136$ (Calcd for $\left.\mathrm{C}_{40} \mathrm{H}_{56} \mathrm{O}_{5}, 616.4125\right)$. $\mathrm{CD}\left(\mathrm{Et}_{2} \mathrm{O}\right): \lambda(\Delta \varepsilon) 240$ $(+2), 250(0), 275(-5.0), 320(0)$.

Acetylation of $2(0.1 \mathrm{mg})$ with $\left(\mathrm{CH}_{3} \mathrm{CO}\right)_{2} \mathrm{O}(5 \mathrm{ml})$ in dry pyridine $(5 \mathrm{ml})$ at room temperature for $60 \mathrm{~min}$ produced a diacetate (yield 90\%) which showed molecular ion at $m / z 700$ by FAB-MS.

Reduction of $2(0.1 \mathrm{mg})$ with $\mathrm{NaBH}_{4}(5 \mathrm{mg})$ in $\mathrm{CH}_{3} \mathrm{OH}(10 \mathrm{ml})$ at room temperature for $30 \mathrm{~min}$ produced a tetrol (yield $80 \%$ ) which showed molecular ion at $m / z 620$ by FAB-MS and absorption maxima $\left(\mathrm{Et}_{2} \mathrm{O}\right)$ at 405,429 , and $465 \mathrm{~nm}$.

Compound (3): Reddish solid. UV-vis: $\lambda_{\max }\left(\mathrm{Et}_{2} \mathrm{O}\right) 455$ and $475 \mathrm{~nm} .{ }^{1} \mathrm{H}-$ NMR $\left(\mathrm{CDCl}_{3}, 300 \mathrm{MHz}\right) \delta: 0.89(3 \mathrm{H}, \mathrm{s}, \mathrm{H}-17), 1.07\left(3 \mathrm{H}, \mathrm{s}, \mathrm{H}-17^{\prime}\right), 1.09$ $\left(3 \mathrm{H}, \mathrm{s}, \mathrm{H}-16^{\prime}\right), 1.23(3 \mathrm{H}, \mathrm{s}, \mathrm{H}-18), 1.47(3 \mathrm{H}, \mathrm{s}, \mathrm{H}-16), 1.57$ (1H, ddd, $J=12.5,4,1.5 \mathrm{~Hz}, \mathrm{H}-2^{\prime}$ eq $), 1.63(1 \mathrm{H}, \mathrm{d}, J=12 \mathrm{~Hz}, \mathrm{H}-2 \mathrm{eq}), 1.68(1 \mathrm{H}, \mathrm{dd}$, $\left.J=12.5,12.5 \mathrm{~Hz}, \mathrm{H}-2^{\prime} \mathrm{ax}\right), 1.71(1 \mathrm{H}, \mathrm{d}, J=12 \mathrm{~Hz}, \mathrm{H}-4 \mathrm{eq}), 1.84(2 \mathrm{H}, \mathrm{ddd}$, $J=12,6,2 \mathrm{~Hz}, \mathrm{H}-2 \mathrm{ax}), 1.90$ (3H, s, H-18'), 1.96 (3H, s, H-19'), $2.04(1 \mathrm{H}$, ddd, $J=12,6,2 \mathrm{~Hz}, \mathrm{H}-4 \mathrm{ax}), 2.24$ (3H, s, H-20), $3.88\left(1 \mathrm{H}, \mathrm{m}, \mathrm{H}-3^{\prime}\right), 3.97$ $\left(1 \mathrm{H}, \mathrm{d}, J=3.5 \mathrm{~Hz}, \mathrm{H}-4^{\prime}\right), 4.41(1 \mathrm{H}, \mathrm{t}, J=6 \mathrm{~Hz}, \mathrm{H}-3), 5.72(1 \mathrm{H}, \mathrm{s}, \mathrm{H}-12), 6.09$ $\left(1 \mathrm{H}, \mathrm{d}, J=16 \mathrm{~Hz}, \mathrm{H}-7^{\prime}\right), 6.10\left(1 \mathrm{H}, \mathrm{d}, J=11.5 \mathrm{~Hz}, \mathrm{H}-10^{\prime}\right), 6.17(1 \mathrm{H}, \mathrm{d}$, $\left.J=16 \mathrm{~Hz}, \mathrm{H}-8^{\prime}\right), 6.41\left(1 \mathrm{H}, \mathrm{dd}, J=14.5,11.5 \mathrm{~Hz}, \mathrm{H}-14^{\prime}\right), 6.44(1 \mathrm{H}, \mathrm{d}$, $J=16 \mathrm{~Hz}, \mathrm{H}-8), 6.44(1 \mathrm{H}, \mathrm{d}, J=11.5 \mathrm{~Hz}, \mathrm{H}-14), 6.50(1 \mathrm{H}, \mathrm{dd}, J=14.5$, $\left.11.5 \mathrm{~Hz}, \mathrm{H}-15^{\prime}\right), 6.57\left(1 \mathrm{H}, \mathrm{dd}, J=14.5,11.5 \mathrm{~Hz}, \mathrm{H}-11^{\prime}\right), 6.64(1 \mathrm{H}, \mathrm{dd}$, $J=14.5,11.5 \mathrm{~Hz}, \mathrm{H}-15), 6.96(1 \mathrm{H}, \mathrm{d}, J=16 \mathrm{~Hz}, \mathrm{H}-7), 7.01(1 \mathrm{H}, \mathrm{s}, \mathrm{H}-10)$. HR-FAB-MS: $m / z 588.3456$ (Calcd for $\mathrm{C}_{37} \mathrm{H}_{48} \mathrm{O}_{6}$ calcd 588.3450).

Acetylation of $3(0.1 \mathrm{mg})$ with $\left(\mathrm{CH}_{3} \mathrm{CO}\right)_{2} \mathrm{O}(5 \mathrm{ml})$ in dry pyridine $(5 \mathrm{ml})$ at room temperature for $60 \mathrm{~min}$ produced a diacetate (yield $90 \%$ ) which showed molecular ion at $m / z 672$ by FAB-MS.

\section{References and Notes}

1) Matsuno T., Hirao S., "Marine Biogenic Lipids, Fats, and Oils," Vol. 1, ed. by Ackman R. G., CRC Press, Boca Raton, Florida, 1989, pp. 251-388.

2) Miki W., Pure Appl. Chem., 63, 141-146 (1991).

3) Nishino H., Tsushima M., Matsuno T., Tanaka Y., Okuzumi J., Murakoshi M., Satomi Y., Takayasu J., Tokuda T., Nishino A., Iwashima A., Anti-Cancer Drugs, 3, 493-497 (1992).

4) Nishino Y., Mutation Res., 402, 159-163 (1998).

5) Tsushima M., Maoka T., Katuyama M., Kozuka M., Matsuno T., Tokuda H., Nishino H., Iwashima A., Biol. Pharm. Bull., 18, 227233 (1995).
6) Khare A., Moss G. P., Weedon B. C. L., Tetrahedron Lett., 14, 39213924 (1973).

7) Hertzberg S., Partali V., Liaaen-Jensen S., Acta Chem. Scand., B42, 495-503 (1988).

8) Campbell S. A., Mallams A. K., Waight E. S., Weedon B. C. L., Barbier M., Lederer E., Salaque A., Chem. Commun., 1967, 941-942 (1967).

9) Matsuno T., Hiraoka K., Maoka T., Nippon Suisan Gakkaishi, 47, $383-390$ (1981)

10) Maoka T., Matsuno M., Nippon Suisan Gakkaishi, 54, 1443-1447 (1988).

11) Maoka T., J. Nat. Prod., 60, 616-617 (1997).

12) Matsuno T., Sakaguchi S., Tetrahedron Lett., 24, 911—912 (1983).

13) Fujiwara Y., Maoka T., Ookubo M., Matsuno T., Tetrahedron Lett., 33, 4941- 4944 (1992).

14) Tsushima M., Maoka T., Matuno T., J. Nat. Prod., 64, 1139-1142 (2001).

15) Maoka T., Hashimoto K., Akimtoto N., Fujiwara Y., J. Nat. Prod., 64, $578-581$ (2001).

16) Matsuno T., Ookubo M., Nishizawa T., Shimizu I., Chem. Pharm. Bull., 32, 4309-4315 (1984).

17) Englert G., "Carotenoids," Vol. 1B, ed. by Britton G., Liaaen-Jensen S., Pfander H., Birkhauser Verlag, Basel, 1995, pp. 147-160.

18) Maoka T., Akimoto N., Fujiwara Y., Hashimoto K., J. Nat. Prod., 67, 115-117 (2004).

19) Britton G., "Carotenoids," Vol. 1B, ed. by Britton G., Liaaen-Jensen S., Pfander H., Birkhauser Verlag, Basel, 1995, pp. 13-62.

20) Buschor D. J., Eugster C. H., Helv. Chim. Acta, 73, 1002-1021 (1990).

21) Deli J., Molnar P., Toth G., Baumeler A., Eugster H., Helv. Chim. Acta, 74, 819-824 (1991).

22) Maoka T., Fujiwara Y., Hashimoto K., Akimoto N., J. Nat. Prod., in press.

23) Liaeen-Jensen S., "Carotenoids," Vol. 3, ed. by Britton G., LiaaenJensen S., Pfander H., Brikhauser Verlag, Basel, 1998, pp. 259-371.

24) Britton G., Liaaen-Jensen S., Pfander H., "Carotenoids Handbook," Birkhauser Verlag, Basel, 2004.

25) Matsuno T., Tani Y., Maoka T., Matsuno K., Komori T., Phytochemistry, 25, 2837-2840 (1986). 